\title{
SOX-2 Mediated Conversion of Astrocyte to Neuron
}

Brady Tucker, Wei Wu, Xiao-Ming Xu

Indiana University School of Medicine, Department of Neuroscience

Background: Axonal regeneration following SCl is crucial if individuals are to avoid permanent neurological damage. A plausible solution to circumvent neuronal death/ axonal degeneration may be in the conversion of ubiquitously distributed astrocytes into neurons. Previous research has shown that the neuronal transcription factor SOX-2 has the potential to convert mature glial cells into neuroblasts (iANBs). Furthermore, iANBs possess the capability to differentiate into functional neurons. Our study employs an in vivo mouse model to demonstrate the functional recovery that SOX-2 mediated conversion of astrocytes to neurons may pose following SCl.

Methods: Our study utilized two populations of mice. The first population received thoracic T10 contusion injuries while the second population received cervical C5 dorsal hemi-sections. Mice were subsequently blindly categorized into groups $A, B$, and $C$ (treatment groups) depending on which injection they would be receiving: LV-hNG2-GFP, LV-hNG2-SOX2, or LV-p75-2.

Following injection, behavioral studies including Hargreaves, roto-rod, grid walk, BMS (contusion mice) and pellet retrieval (DH mice) were performed to assess functional recovery at 2 week intervals.

Results: The astrocyte conversion to neuron project spans more than 16 weeks. Here we present data obtained in the first eight weeks which includes behavioral analyses of contusion injury mice. Currently grid walk, BMS, and Hargreaves testing show similar trends in spontaneous recovery however no significant difference is observed between the independent injection groups. On the contrary roto-rod analyses showed injection group $\mathrm{C}$ had a significantly lower latency to fall time in comparison to groups A and B six weeks post injection (wpi).

Conclusion: The treatments are hypothesized to not take effect until approximately 8 wpi thus we expect subsequent behavioral testing to reveal significant differences between treatment groups, ultimately taking one step closer towards therapeutic intervention following traumatic SCl. 